\title{
Research on the properties of Emulsified Asphalt Absorption and attenuation of microwave
}

\author{
Rongwang Guo, Xiang Wu, Hui Chen \\ Academy of Engineer Corps, China
}

Keywords: Emulsified asphalt, Absorption, Attenuation, Microwave .

\begin{abstract}
Gauging the emulsified asphalt with gravel or machine sand, lime and paint, can formate emulsified asphalt slurry seal. In the military field, emulsified asphalt materials can be used to constructe the false asphalt pavement. This paper mainly analyses the choice and technical requirements of materials, discusses concrete construction steps.
\end{abstract}

\section{Introduction}

Emulsified asphalt is a kind of OW or WO asphalt emulsion, which created by heating asphalt until melted, mechanical trituration, and making the asphalt with small droplets dispersed in aqueous solution containing asphalt emulsifier evenly. Emulsified asphalt mainly consists of three parts, they are asphalt, water and emulsifier, besides, it maybe also includes a few additives, such as stabilizer, special auxiliary, modifier and so on. Emulsified asphalt has good absorption properties of carbon fiber, besides strong adhesion, fine waterproof, good durability Etc. So, it can be applied in the absorption and attenuation of microwave.

\section{Absorption and attenuation of microwave by emulsified asphalt}

\section{Design of absorbing materials}

Good absorbing materials must have two conditions: First, when the electromagnetic waves transmit to the absorbing materials surface, it can enter the interior of the absorbing materials to the utmost, so it requires to consider the characteristics of wave impedance matching fully in the process of designing materials; Second, when the electromagnetic waves transmit to the interior of the absorbing materials, it requires the absorbing materials to absorb and attenuate the electromagnetic waves effectively, that is the electromagnetic loss, so it requires to consider the characteristics of the electromagnetic loss of the materials.

Design of absorbing materials needs to void reflection as far as possible, one of the primary factors is the matching problem of wave impedance. When the electromagnetic transmits from free space with $Z_{0}$ impedance to absorbing interface with $Z_{1}$ impedance, one part is reflected, the other part enter the absorbing materials. The reflectance $\mathrm{R}$ of absorbing materials can be formulated as follows:

$$
\begin{aligned}
& R=\left(Z_{0}-Z_{1}\right) /\left(Z_{0}+Z_{1}\right) \\
& Z_{I}=\left(\mu_{\mathrm{i}} / \varepsilon_{\mathrm{i}}\right)^{1 / 2}, \quad I=0,1
\end{aligned}
$$

As the formulas: $\mu$ and $\varepsilon$ are permeability and dielectric constant of the materials, if it wants to acheieve complete no reflection, that is to say $R=0$, it requires $Z_{0}=Z_{1}$, but if $Z_{0}$ is a vaccum, then $\mu_{0}=\varepsilon_{0}$.

So, hign-performance absorbing materials requires that both of them keep equal approximately in the frequency range as wide as possible. Another factor of designing absorbing materials is improving electromagnetic loss, to translate the electromagnetic into heat or any other energy, so that the electromagnetic can be absorbed by medium at the utmost. The complex dielectric constant $\mu$ and complex permeability $\varepsilon$ of absorbing materials can be formulated as follows:

$$
\mu=\mu^{\prime}-\mathrm{i} \mu{ }^{\prime \prime}
$$




$$
\varepsilon=\varepsilon^{\prime}-\mathrm{i} \varepsilon^{\prime \prime}
$$

As the formulas: $\varepsilon^{\prime}$ and $\mu^{\prime}$ are variables of polarization and magnetization of absorbing materials under the action of electric or magnetic field. $\varepsilon^{\prime \prime}$ is a measure of the loss caused by electric dipole rearrangement of materials in the external electric field, $\mu^{\prime \prime}$ is a measure of the loss caused by magnetic dipole rearrangement of materials in the external magnetic field. On the basis of electrodynamics, $\varepsilon^{\prime \prime}$ and $\mu^{\prime \prime}$, which are the imaginary parts of $\varepsilon_{\text {and }} \mu$, characterize the absorption of electromagnetic waves for medium. According the definition of physics, there is:

Electric loss tangent

Magnetic loss tangent

$$
\tan \partial_{E}=\varepsilon^{\prime \prime} / \varepsilon^{\prime}
$$

$$
\tan \partial_{M}=\mu^{\prime \prime} / \mu^{\prime}
$$

The formulas above indicates, the lager the loss of material, the bigger $\varepsilon^{\prime \prime}$ and $\mu^{\prime \prime}$ are required, and the wave absorbing properties is better. Increasing wave absorbing properties of medium requires to increase $\varepsilon^{\prime \prime}$ and $\mu^{\prime \prime}$ by increasing conductivity, polarization and magnetic friction, and satisfying the impedance matching conditions. For single component medium, it is hard to satisfy impedance matching and strong absorption simultaneously, the material with $\varepsilon=\mu$ is difficult to find too. Increasing polarization friction is a important method to increase the loss, but when the polarization friction acheives the standard of metal, reflectivity $\mathrm{R}$ closes to one, it will be far from matching. According the analysis above, choosing carbon fiber as absorbing material is more appropriate.

\section{Carbon fiber}

Carbon fiber is a fibrous carbon material formated by heating organic fiber or low molecular hydrogen gas, is incomplete graphite crystal arranged along fibers in axial direction, it's carbon content is above $90 \%$. With the increase of temperature, the carbon fiber structure transfers from trubostratic structure to three-dimensional graphite structure, space reduces, conductivity increase, it is easy to form strong reflectors of radar wave. Carbon fiber treated by low temperature, it's structure is loose, it is an absorber of electromagnetic wave and a electric loss material with good electrical performance. So, only carbon fiber after special treatment can absorb radar wave. Continuous carbon fiber can strongly reflect radar wave, mainly because electromagnetic field formates large continuous conduction current in carbon fiber.

For the moment, absorbing mechanism of short carbon fiber in matrix has two explanations: One is thinking short carbon fiber has an action of resonant in absorbing materials. There is a quasi steady induced field in the near field of short carbon fiber, the field arouses dissipation current, and the dissipation current reduces by the action of surrounding matrix, and it transmits radar wave energy to any other energies, mainly heat. Another is thinking short carbon fiber can be regarded as doublet in absorbing materials containing short carbon fiber. Short carbon fiber doublet can creat dissipation current in the action of electromagnetic field, and the dissipation current reduces by the action of surrounding matrix, and it transmits radar wave energy to any other energies. Carbon fiber minicrystal prefers orientation along the axial direction in the progress of growth, for the obviously multi-directional of electromagnetic wave, the magnetic and electric loss of electromagnetic wave can achieve the desired level. Otherwise, density of pitch based carbon fiber only has one to two grams per cubic centimetres, it is easy to manufacture lightweight composite materials, achieve the requires of high efficient and broadband absorption. Carbon fiber minicrystal prefers orientation along the axial direction in the progress of growth, for the obviously multi-directional of electromagnetic wave, it can achieve optimizing material absorption performance by designing arrangement of carbon fiber in the matrix. 


\section{Manufacturing pitch based carbon fiber}

i) Precision manufacturing of raw asphalt

Asphalt, especially coal-tar pitch, usually contains free carbon and solid impurity, they can plug the spinning hole in the process of spinning, fine particles left in the fiber become the fracture origin of carbon fiber. So, it must finely product the asphalt to remove the impurities above. Common method is adding certain solvent in asphalt, and heating the asphalt up to $100^{\circ} \mathrm{C}$, then filtering with stainless steel mesh or heat resistant feberglass in the protection of nitrogen to avoid oxidizing.

ii) Modulation of emulsified asphalt

Modulation of asphalt has three purposes: one is transfering asphalt to cold emulsified asphalt by using emulsifier; another is removing light components in asphalt, to prevent the generation of bubbles made fracture filamentation in spinning process; the third is epuidistributing moleculars by improving the softening point. Modulation is to manufacturing spinning emulsified asphalt by thermal condensation, hydropretreating and solvent extraction.

iii) Preparation of emulsified asphalt carbon fiber

The melt spinning of emulsified asphalt is different from common macromolecule, it can't draft any more after solidifying in a very short time, and the fiber manufactured is very fragile, therefore, it requires the diameter of fiber is below 15 in the process of spinning, to increase the strongth of carbon fiber. The methods of carbon fiber spinning mainly include extrusion, centrifugal, meltblowing and eddy-current. Extrusion method is pressing the high temperature melting emulsified asphalt in the spinneret, and then squeeze into filaments. Centrifugal method is pouring the high temperature melting emlsified asphalt into the centrifugal drum with high-speed rotation, and then being thrown out into cellosilk by the action of centrifugal force. Melt-blowing method is sending $\mathrm{t}$ into the spinneret, and then being cooled by high gas flow into cellosilk after being squeezed from the holes. Eddy-current method is the high temperature melting emlsified asphalt being blowed-out and drafted by thermal current along the direction of its flow, the fiber spinned is curly unregularly.

iv) Pre-oxidation and no-melting treatment of emulsified asphalt carbon fiber

It needs to remove carbon atoms from emulsified asphalt carbon fiber by carbonization, to play inherent properties of carbon elements. Because of the solubility and stickiness of emulsified asphalt, it can't formate carbon fiber monofilament at the beginning of heating, so it must conduct pre-oxidation firstly. Otherwise, pre-oxidation can improve mechanical property, improve tensile strength. Pre-oxidation includes gas phase method and liquid phase method. The oxidants of gas phase method are usually air, N02, S03, O3 and oxygen enriched gas. The oxidants of liquid phase method are usually hydrogen nitrate, sulphuric acid, mineral chameleon and hydrogen peroxide. The temperature of oxidation is usually between $200-400^{\circ} \mathrm{C}$. It requires to oxidate evenly in the process of oxidation, and shouldn't formate the structure with lower edge of the center.

v) Carbonization and graphitizing treatment of emulsified asphalt carbon fiber

Take emulsified asphalt carbon fiber after pre-oxidation to be in inert atmosphere for carbonization and graphitizing treatment, improve the final mechanical properties. Carbonization treatment is at about $1200{ }^{\circ} \mathrm{C}$, graphitizing treatment is at about $3000{ }^{\circ} \mathrm{C}$. In the process of carbonization treatment, polycondensation is produced between unimolecules, with the reaction of dehydrogenation, demethanization and degydration. As the carbon atoms are eliminated continuously, carbon content of fiber after carbonization is up to $92 \%$, the inherent properties of the carbon is improved, filaments' tensile strength and modulus increase.

vi) Subsequent treatment of emulsified asphalt carbon fiber

In order to further improve the affinity and the cohesive force between emulsified asphalt carbon fiber and composite matrix, it needs surface treatment of emulsified asphalt carbon fiber, to remove surface impurities, and formate micropores on the surface, increase the surface energy. The methods are air oxidation, liquid phase oxidation and so on. 


\section{Conclusion}

Adding the absorbing material in emulsified asphalt can strength the properties of absorption and attenuation of microwave, by the use of strong inclusive and high absorption rate in carbonized material of emulsified asphalt.

\section{References}

[1] International Slurry Surfacing Association,Recommended Performance Guidelines for Emulsified Asphalt Slurry Seal Surfaces,A105（revised） May 2005

[2] Guowei Yang, etc. Preparation and absorbing properties of hollow asphalt base carbon fiber. Progress of chemical industry. No.8, 2009: 1400 1404

[3] Pengyuan Yang, etc. Research on asphalt base carbon fiber composite wave absorbing materials. Chemical Journal. No.3, Mar.2009: 53 55 